\title{
THE FIRST SPECTROSCOPICALLY RESOLVED SUB-PARSEC ORBIT OF A SUPERMASSIVE BINARY BLACK HOLE
}

\author{
E. Bon ${ }^{1,2}$, P. Jovanović ${ }^{1,2}$, P. Marziani ${ }^{3,4}$, A. I. Shapovalova ${ }^{5}$, N. Bon ${ }^{1,2}$, \\ V. Borka Jovanović ${ }^{2,6}$, D. Borka ${ }^{2,6}$, J. SulentiC ${ }^{4}$, and L. Č. Popović ${ }^{1,2}$ \\ ${ }^{1}$ Astronomical Observatory, Volgina 7, 11060 Belgrade, Serbia \\ ${ }^{2}$ Isaac Newton Institute of Chile, Yugoslavia Branch, Belgrade, Serbia \\ ${ }^{3}$ INAF, Osservatorio Astronomico di Padova, Padova, Italy \\ ${ }^{4}$ Instituto de Astrofísica de Andalucía, CSIC, Apdo. 3004, E-18080 Granada, Spain \\ ${ }^{5}$ Special Astrophysical Observatory of the Russian AS, Nizhnij Arkhyz, Karachaevo-Cherkesia 369167, Russia \\ ${ }^{6}$ Atomic Physics Laboratory (040), Vinča Institute of Nuclear Sciences, University of Belgrade, P.O. Box 522, 11001 Belgrade, Serbia \\ Received 2012 June 15; accepted 2012 September 19; published 2012 October 26
}

\begin{abstract}
One of the most intriguing scenarios proposed to explain how active galactic nuclei are triggered involves the existence of a supermassive binary black hole $(\mathrm{BH})$ system in their cores. Here, we present an observational evidence for the first spectroscopically resolved sub-parsec orbit of a such system in the core of Seyfert galaxy NGC 4151. Using a method similar to those typically used for spectroscopic binary stars, we obtained radial velocity curves of the supermassive binary system, from which we calculated orbital elements and made estimates about the masses of the components. Our analysis shows that periodic variations in the light and radial velocity curves can be accounted for by an eccentric, sub-parsec Keplerian orbit with a 15.9 year period. The flux maximum in the light curve corresponds to the approaching phase of the secondary component toward the observer. According to the obtained results, we speculate that the periodic variations in the observed $\mathrm{H} \alpha$ line shape and flux are due to shock waves generated by the supersonic motion of the components through the surrounding medium. Given the large observational effort needed to reveal this spectroscopically resolved binary orbital motion, we suggest that many such systems may exist in similar objects even if they are hard to find. Detecting more of them will provide us with insight into the $\mathrm{BH}$ mass growth process.
\end{abstract}

Key words: black hole physics - galaxies: active - galaxies: interactions - galaxies: Seyfert - quasars: individual (NGC 4151) - shock waves

\section{INTRODUCTION}

Different mechanisms have been proposed to explain broadline region (BLR) variability in active galactic nuclei (AGNs; see, for e.g., Gaskell \& Klimek 2003; Gaskell 2009 and references therein). One of the most interesting possibilities involves the existence of a binary system in the core (see, for e.g., Komossa 2006; Bogdanović et al. 2008; Gaskell 2009; Tsalmantza et al. 2011; Eracleous et al. 2012; Popović 2012, and references therein). If black hole $(\mathrm{BH})$ mass grows via major mergers, then we might expect to see the signature of a binary BH in some or many active galaxies, if the merger process involves slow coalescence of the two components.

NGC 4151, one of the best-studied Seyfert galaxies, shows a complex sub-parsec structure. Studies at radio, visible, and $\mathrm{X}$-ray wavelengths indicate violent processes including ejection of gas. Detection of outflow scales ranging from several hundred parsecs down to sub-parsec scales reveals collimated jets and biconical outflows (see, e.g., Kraemer et al. 2001; Mundell et al. 2003; Kraemer et al. 2008; Mundell et al. 1999 and references therein). Violent variability in this AGN has been monitored over many years (Shapovalova et al. 2008). Earlier work showed variation in the wings of the $\mathrm{C}$ Iv line (Ulrich et al. 1985) that were associated with two localized emitting regions with line-of-sight velocities of -6100 and $+8500 \mathrm{~km} \mathrm{~s}^{-1}$ with respect to the systemic velocity. These localized regions were thought to be the possible signatures of orbiting matter or of a two-sided jet. A radio jet or highly inclined disk (length $\sim 1 \mathrm{pc}$ ) aligned perpendicular to the arcsec-scale radio jet was imaged at $6 \mathrm{~cm}$ (Ulvestad et al. 1998). Flux variations in the optical domain have been monitored over a much longer time (over 100 years; see, for e.g., Guo et al. 2006; Oknyanskij \& Lyuty 2007 and references therein). Optical and UV emission is unresolved so clues about the central structure come mainly from analysis of the broad emission line profile. Reverberation mapping studies (see, for e.g., Onken et al. 2007; Shapovalova et al. 2008 and references therein) suggest a very compact BLR with broad Balmer lines responding to continuum changes within a few days. Many authors claim to find long-term periodicities in the flux variations of NGC 4151 (Longo et al. 1996) of e.g., 15.8 years (Oknyanskij et al. 1978; Oknyanskij \& Lyuty 2007; Guo et al. 2006; Pacholczyk et al. 1983; Chuvaev et al. 2008). Compared to Seyfert 1s, NGC 4151 has a harder X-ray spectrum and is also similar to BH binaries in the hard state (Lubinski et al. 2010).

In this paper, we analyze the $\mathrm{H} \alpha$ line shape and flux variability in NGC 4151 during a period of more than 20 years in order to investigate its BLR structure. The possibility of periodicity in the line profile variations leads us to interpret it in terms of binary system orbital motion at sub-parsec scales in the center of NGC 4151.

The paper is organized as follows: in Section 2, we briefly describe our data set, method, and analysis used to detect periodicity in both the line flux and the shape of the profiles. In Section 3, we discus possible scenarios for the obtained periodic variations, and give evidence of a sub-parsec supermassive binary BH system in the core of NGC 4151. In Section 4, we discuss alternative models, and the implications of our results. Finally, in Section 5, we summarize our results and give conclusions. 

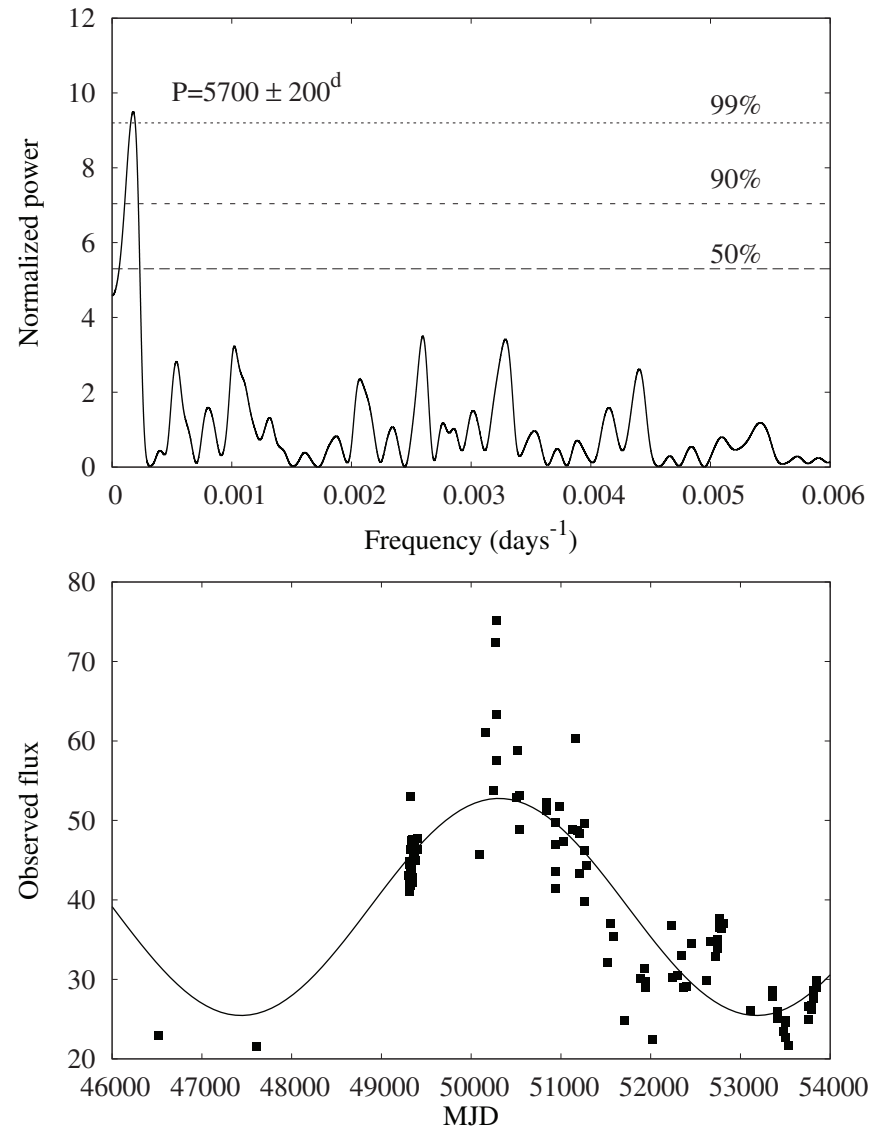

Figure 1. Lomb-Scargle normalized periodograms of the light curves for total flux (top) and the corresponding sinusoidal fit (bottom).

\section{ANALYSIS}

We analyzed 115 spectra of the $\mathrm{H} \alpha$ line profile in NGC 4151 covering more than 20 years. Most of the spectra were obtained during a period of 11 years from 1996 to 2006 (Shapovalova et al. 2008, 2010). This data set was supplemented with: (1) AGN watch spectra observed for three months beginning 1993 November 14 (Kaspi et al. 1996) with the $1 \mathrm{~m}$ Wise Telescope; ${ }^{7}$ (2) one spectrum from the Asiago $1.82 \mathrm{~m}$ Ekar telescope of the Padova Astronomical Observatory (1989 March 24), which is equipped with a Boller and Chivens spectrograph and a $600 \mathrm{gr} \mathrm{mm}^{-1}$ grating, yielding a resolution of 4-5 ^ FWHM, with PA $=90$, at the Cassegrain focus; and (3) a spectrum from 1986 March 29 (Ho et al. 1995). ${ }^{8}$ All spectra were reduced to a common resolution of $15 \AA$ (about $\sigma=340 \mathrm{~km} \mathrm{~s}^{-1}$ ). The continuum was removed by subtracting a linear fit between 6265 and $6830 \AA$. All spectra were scaled to the flux of [O I $] \lambda 6300$, which is assumed constant at all epochs.

\subsection{Flux Variations}

We derived $\mathrm{H} \alpha$ light curves for the total flux (see Figure 1) as well as for the partial flux in $1000 \mathrm{~km} \mathrm{~s}^{-1}$ velocity bins (Figure 2). We analyzed the light curves using Lomb-Scargle spectral analysis (Lomb 1976; Scargle 1982) procedures.

The Lomb-Scargle normalized periodogram (Lomb 1976; Scargle 1982; Press et al. 1996) is a powerful tool for finding

\footnotetext{
7 The AGN watch data could be obtained in digital format from the following link: http://www.astronomy.ohio-state.edu agnwatch/data.html

8 The spectrum from Ho et al. (1995) is public and could be obtained in digital format from the NED: http://ned.ipac.caltech.edu
}

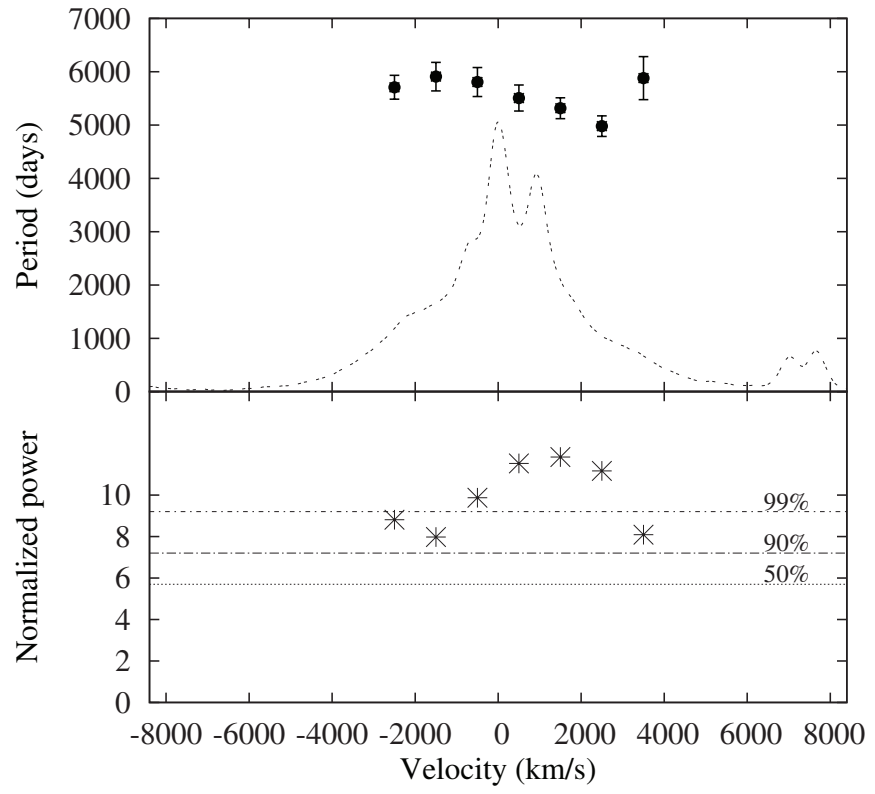

Figure 2. Period (filled circles with error bars) obtained for each $1000 \mathrm{~km} \mathrm{~s}^{-1}$ light curve of $\mathrm{H} \alpha$ line profile and their normalized powers (asterisks). Horizontal straight lines represents $50 \%, 90 \%$, and $99 \%$ normalized power significance levels.

and testing the significance of weak periodic signals embedded in otherwise random and unevenly sampled observations. The significance level (i.e., the probability that the data contain a periodicity) can be tested fairly rigorously by the statistic $1-p$, where $p$ is the so-called false alarm probability. This estimates the probability that the peak occurred in the presence of pure independently and normally (Gaussian) distributed noise. A small value for the false alarm probability indicates significant periodic signal. Resultant periodicities were subsequently tested by fitting with sine functions (see Figure 1, bottom).

Since we detected the periodicity in the $\mathrm{H} \alpha$ line light curve (see Figure 1), we tried to identify the parts of the line profiles that are most affected by those periodical variations. For that purpose, we analyzed the light curves for each $1000 \mathrm{~km} \mathrm{~s}^{-1}$ radial velocity bin of $\mathrm{H} \alpha$ spectra, with a significance level above $90 \%$ (see Figure 2). Since we detected periodicity in certain light curves (mainly in the core of the line for the velocity bins between -3000 and $4000 \mathrm{~km} \mathrm{~s}^{-1}$ ), we tried to uncover the possible mechanisms that could produce such flux variations. For that purpose, we tested many different line decomposition models, taking into account the parts of the line where we detected significant periodicity.

\subsection{Radial Velocity Curves}

We used Gaussian analysis to measure profile shape variations. This was accomplished by fitting each $\mathrm{H} \alpha$ profile with the sum of Gaussians assuming some constraints. The intensity ratio of narrow components $(\mathrm{H} \alpha$ line, $[\mathrm{O} \mathrm{I}] \lambda \lambda 6300,6364$, [N II] $\lambda \lambda 6548,6584$, [SII] $\lambda \lambda 6717,6731$, and $\mathrm{He}$ I $\lambda 6678)$ was modeled using spectra obtained during the minimum activity stage (2005 May 12). They were fitted with the sum of Gaussian profiles assuming that the intensity ratios of the narrow lines did not change during the monitoring period. The widths of the narrow lines were assumed equal during each fit.

The broad $\mathrm{H} \alpha$ profile was fit together with narrow lines. We tested a number of models, including those with more than one Gaussian component (see, for e.g., Shen \& Loeb 2010), and 
analyzed the light curves of the component fluxes as well as the radial velocity curves of each component during the monitoring period. The choice of component widths was not arbitrary. We started from the decomposition proposed in Sulentic et al. (2000) and Marziani et al. (2010). We noticed that a two-component model (Bon et al. 2009, 2006) consisting of very broad (covering the far wings) and broad (covering the line core) components could not properly explain an intermediate width feature visible on the $\mathrm{H} \alpha$ wing $\left(\sigma=600 \mathrm{~km} \mathrm{~s}^{-1}\right)$. R and VBLR components are typical of Population B AGNs (Sulentic et al. 2000; Marziani et al. 2010), while displaced subpeaks have been occasionally observed in this class (Zamfir et al. 2010). The intermediate width subpeak was observed on both the blue and red wings of $\mathrm{H} \alpha$ at different epochs but never at the same time, leading us to interpret it as a single emitting component $\left(\sigma=600 \mathrm{~km} \mathrm{~s}^{-1}\right)$.

We then tried many fits with different widths for each of the three $\mathrm{H} \alpha$ components and adopted a model that consists of: (1) a very broad component (VBC) corresponding to the far wings $\left(\sigma=3400 \mathrm{~km} \mathrm{~s}^{-1}\right)$, (2) a central broad component (CBC) corresponding to the "classical" broad core of the line $\left(\sigma=1700 \mathrm{~km} \mathrm{~s}^{-1}\right)$, and (3) an intermediate width component (bump) appearing on either the blue or red wing of the $\mathrm{H} \alpha$ profile $\sigma=600 \mathrm{~km} \mathrm{~s}^{-1}$ ). The choice of Gaussian component widths were made in such a way that each component width differs for at least two times, providing a clear identification of each component. We fit the $\mathrm{H} \alpha$ profile using three components with fixed widths. The CBC shift was fixed to the shift of the narrow lines.

To secure the identification of the Gaussian components, we adopted the model with three Gaussians with several important limitations according to the shapes of profiles. We measured the width of the bump on profiles where it could be clearly isolated from other components. We found that the width of the bump's Gaussian is around $\sigma=600 \mathrm{~km} \mathrm{~s}^{-1}$. We also tested the widths of two other components and found that the Gaussian suitable to cover the central part of the line profiles (CBC) needed to be at least twice the width of the bump's Gaussian. Also, we fixed the shift of the $\mathrm{CBC}$, since we noticed that the shift of this component was showing very small variations (smaller than the estimated errors for this parameter), since the very broad wings (which were significantly building up in many profiles) could be covered only with a very broad Gaussian (at least twice the width of the $\mathrm{CBC}$ ). We noticed that when the bump component is present on the red side of the profile, it was appearing either as a separated peak, or as a feature that was making the red side of the CBC much steeper than the regular shape of the Gaussian component that fit the core of the line (CBC). When the bump component was appearing on the blue side, it was just changing the steepness of the blue side of the profile, making it much steeper than the shape of the Gaussian of the CBC. In this way, including the bump component was justified in our model. Similarly, it was necessary to introduce the VBC Gaussian to fit the far wings in some profiles that also could not be reproduced by the other two components.

In the case where we vary the intensities of the CBC and the VBC, the position of the bump would shift, but for the solutions where the fit looks reasonable, the shifts of the bump are smaller than the estimated errors. For example, if intensities are changed for less than $5 \%$, then the shift of the bump is changed, but stays inside the error interval, while reduced $\chi^{2}$ increases for more than an order of magnitude. If the variations of intensities are even larger, then the fit becomes unreasonable and do not follow the shape of the profile.
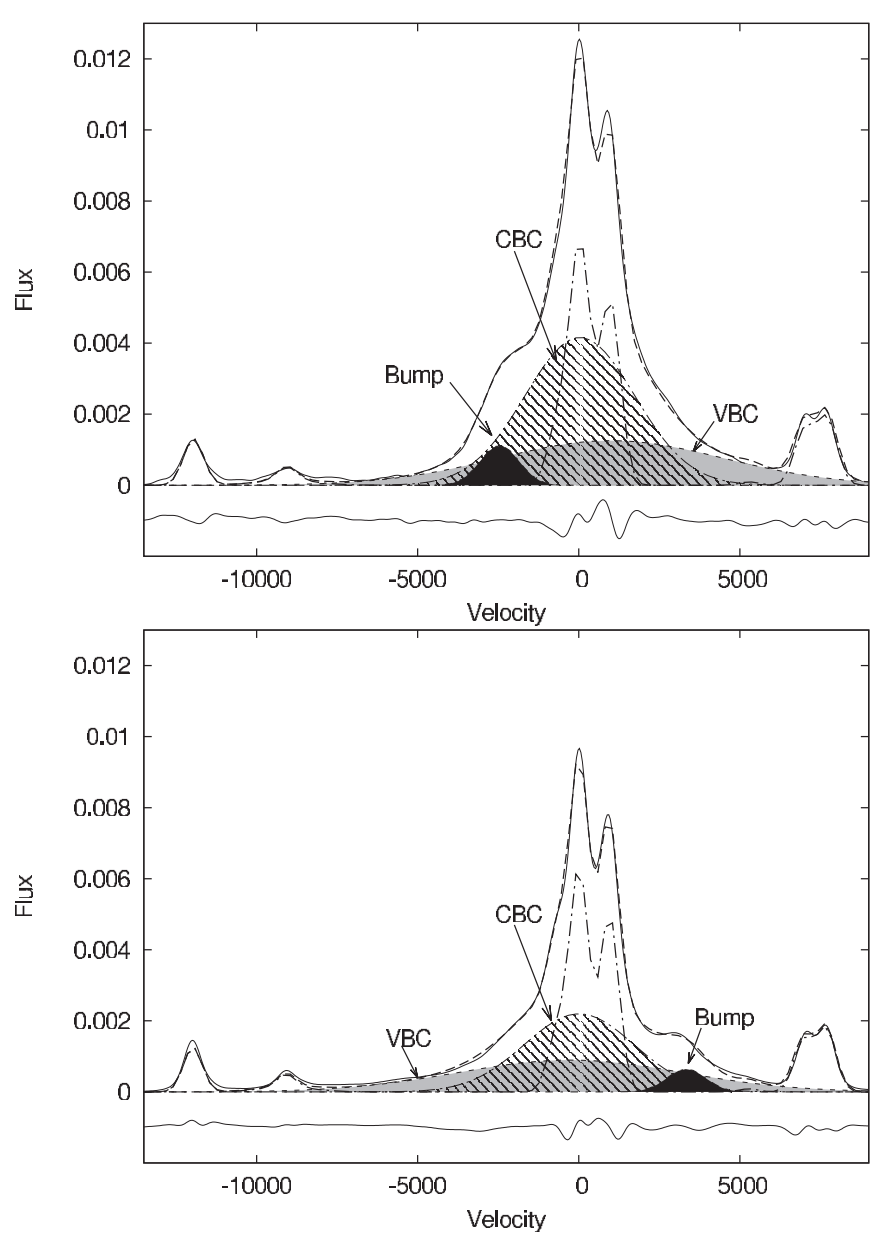

Figure 3. Examples for the best fit (dashed line) of the $\mathrm{H} \alpha$ line profile (solid line) for two epochs: MJD 51203 (up) and 52621 (down). Three Gaussian components, VBC ( $\sigma=3400 \mathrm{~km} \mathrm{~s}^{-1}$, shaded with gray), CBC ( $\sigma=$ $1700 \mathrm{~km} \mathrm{~s}^{-1}$, shaded with line pattern $)$, and the bump $\left(\sigma=600 \mathrm{~km} \mathrm{~s}^{-1}\right.$, shaded with black) component, are fitted in the broad profile. The dash-dotted line represents the narrow line template. The panel at the bottom of the plots represents residuals of the fit.

Examples of fits for two epochs are shown in Figure 3. We noticed that the $\mathrm{CBC}$ and $\mathrm{VBC}$ give major contributions to the total flux of the line, and hence their light curves follow the total $\mathrm{H} \alpha$ line light curve. On these light curves (see Figure 4, bottom), one can identify one dominant peak in the interval of the Modified Julian Date (MJD) between 50000 and 51000 MJD and another smaller peak between 52500 and 53000 MJD. Since we found similar behavior in the light curves of all components, as well as in the continuum and the $\mathrm{H} \alpha$ line, we concluded that the flux variations are real in all components and are not caused by of the Gaussian fitting procedure. More spectra from each observational campaign with corresponding fits are presented in Figures 5-10.

Bogdanović et al. (2008) made simulations of $\mathrm{H} \alpha$ emission line profiles in the case of a supermassive binary BH system with masses of $10^{7}$ and $10^{8} M_{\odot}$ and the following orbital elements: 15.7 year period and semimajor axis of $a=0.01 \mathrm{pc}$. It is not that obvious, but one can see in their Figure 18 that the emission line profiles show a bump peak that appears on the blue side of the profile for the first part of the orbital period. In the second part of the orbital period, the bump peak starts to appear on the red side of the profile. Also, one can see that the central peak as well as the wider structure (VBC) is present in most of the profiles, even though we approximated it with a Gaussian instead of the 


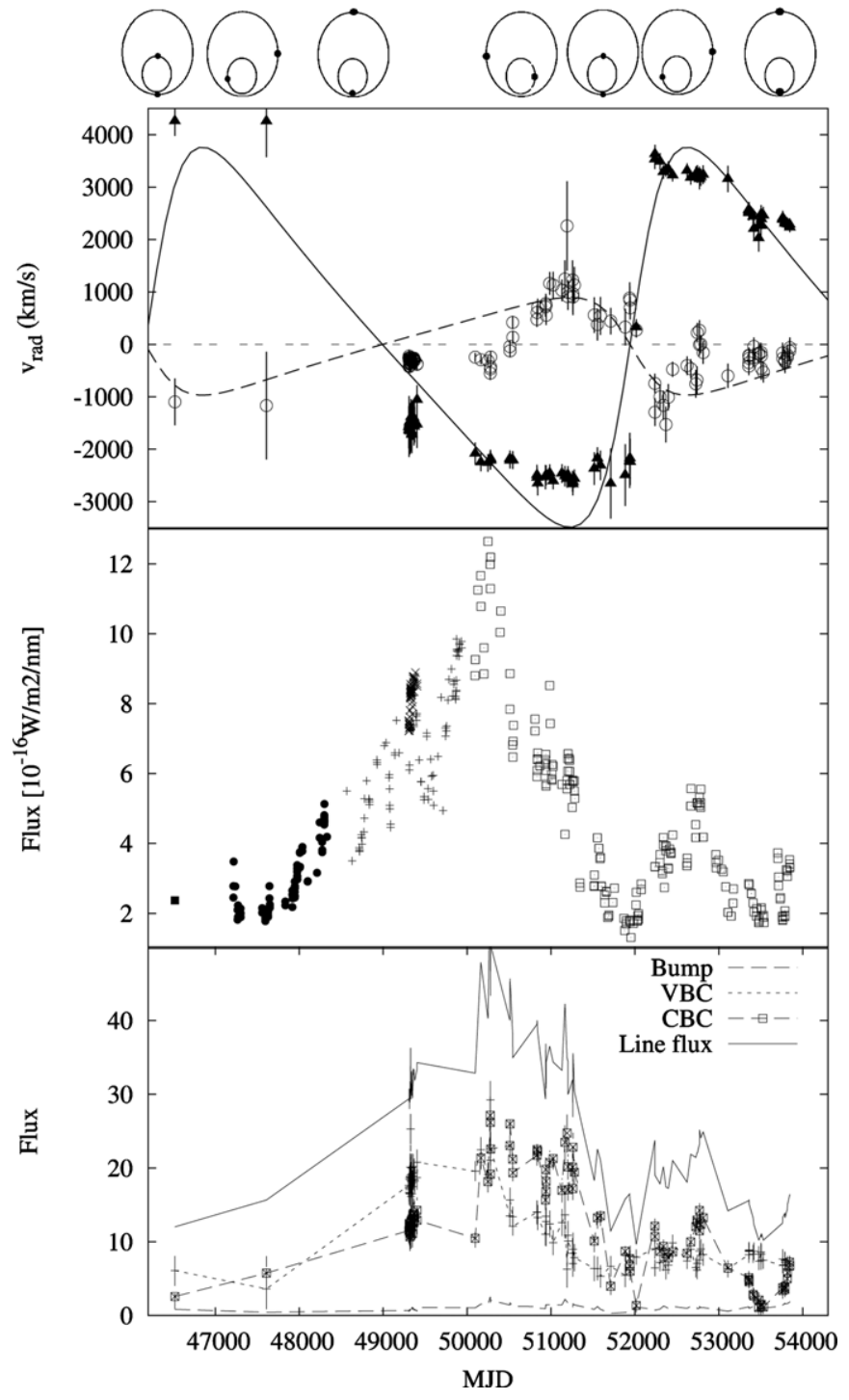

Figure 4. Keplerian orbits of a supermassive black hole binary. Top: radial velocity curves of the bump (open triangles with $y$ error bars) and VBC (open circles with $y$ error bars) obtained from Gaussian decomposition of the broad $\mathrm{H} \alpha$ line, as well as fitted radial velocity curves for orbits of both components (solid and dashed lines). Middle: continuum flux light curves for NGC 4151 at $512.5 \mathrm{~nm}$, compiled from Malkov et al. (1997; pluses), Kaspi et al. (1996; crosses), Shapovalova et al. (2008; open squares), as well as at $656.3 \mathrm{~nm}$ from Sergeev (1994; filled circles). Bottom: light curves of the relative fluxes (normalized to the flux of O I line) with $y$ error bars of VBC (doted line), CBC (dashed line with open squares), bump component (dashed line), and the $\mathrm{H} \alpha$ line (solid line). Illustration of the corresponding orbital phases are presented above the radial velocity curve panel, assuming counterclockwise direction of each black hole motion and the direction toward the observer below the plots.

more complex profile as seen in this figure (probably a disk-like emission profile). In our case, there was no significant difference in the radial velocity curves and light curves in the case of fitting using a VBC Gaussian instead of a VBC disk profile.

We analyzed radial velocity variations in the $\mathrm{VBC}$ and bump components. We also fit radial velocity curves assuming Keplerian orbits using the "velocity" code (Rainer 1988). The uncertainty in the position of the bump was estimated to be $\pm 200 \mathrm{~km} \mathrm{~s}^{-1}$ (when detected on the blue side) and $\pm 70 \mathrm{~km} \mathrm{~s}^{-1}$ (on the red) at $2 \sigma$ confidence level $(P=0.99)$. Estimated uncertainties for the radial velocity of the VBC were around $\pm 300 \mathrm{~km} \mathrm{~s}^{-1}$. These errors were set by the $\chi^{2}$ behavior when

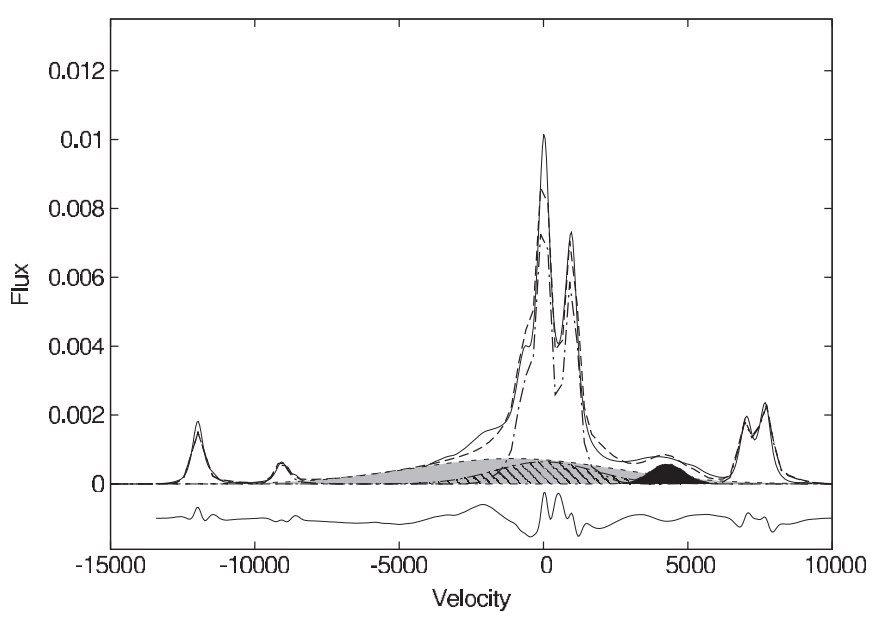

Figure 5. Same as in Figure 3 but for the spectrum from Ho et al. (1995) (epoch MJD 46518.5).

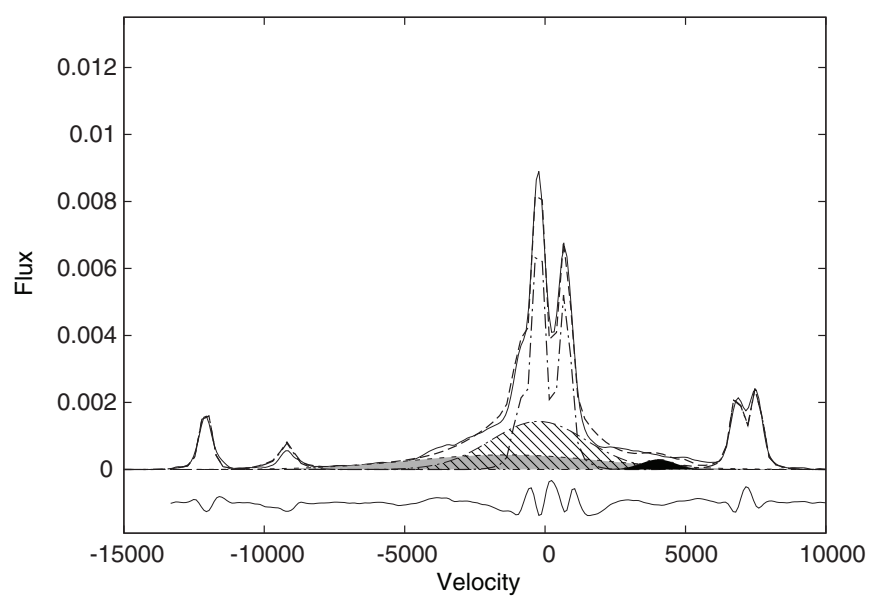

Figure 6. Same as in Figure 3, but for the spectrum obtained on Asiago $1.8 \mathrm{~m}$ Ekar telescope (epoch MJD 47609.529).

varying only the displacement from the best-fit radial velocity and are regarded as minimum uncertainties.

To test the influence of the fitting procedure on the light curves and radial velocity curves, we varied the widths of each component. For the bump, we found that in some cases the fit becomes unreasonable if we change its width by more than $100 \mathrm{~km} \mathrm{~s}^{-1}$. For the change in the widths of the VBC and CBC, the radial velocity curves of the bump component did not change much (a few times less than the estimated errors), even though the VBC width was varied from 3000 to $4500 \mathrm{~km} \mathrm{~s}^{-1}$, and CBC from 1500 to $2000 \mathrm{~km} \mathrm{~s}^{-1}$. We noticed that for the broader VBC, the amplitude of the radial velocity curve of that component became larger, but kept the same shape and behavior, and hence led to similar orbital parameters. However, in that case, the obtained mass ratio between the secondary (bump) and primary (VBC) will vary between 0.2 (for $\sigma_{\mathrm{VBC}}=3000 \mathrm{~km} \mathrm{~s}^{-1}$ ) and 0.6 (for $\sigma_{\mathrm{VBC}}=4500 \mathrm{~km} \mathrm{~s}^{-1}$ ). Therefore, this method gives more reliable estimations of the orbital elements than those for the mass ratio.

We are aware that when the bump is close to the line core (at $v_{r} \sim 0$ ), its position is well constrained because of the model we build with the three symmetric Gaussians, since the bump is overwhelmed by the $\mathrm{BC}$ and $\mathrm{VBC}$ emission so as to become not visually identifiable. As the bump radial velocity changes sign rather suddenly, this condition occurs only for a few epochs. 


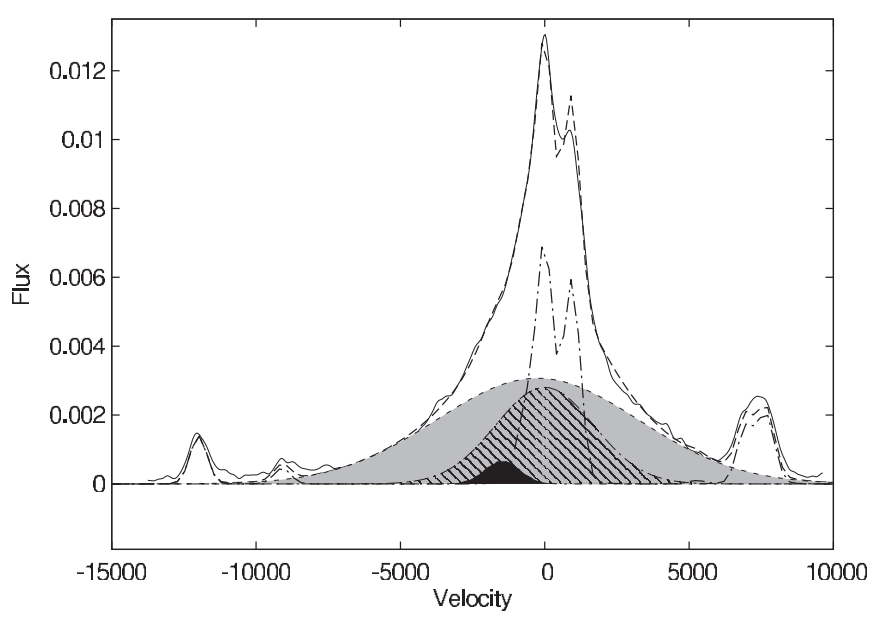

Figure 7. Same as in Figure 3, but for the spectrum obtained during the AGN watch program (Kaspi et al. 1996; epoch MJD 49327.577).

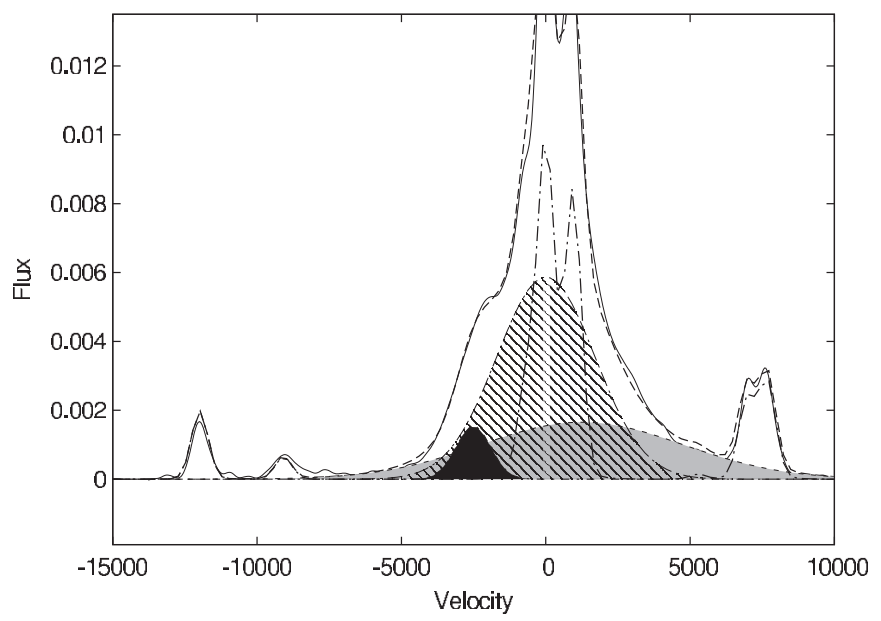

Figure 8. Same as in Figure 3, but for the spectrum from the set of Shapovalova et al. (2008, 2010; epoch MJD 51166.656) with the bump component positioned on the blue part of the spectrum.

\section{RESULTS}

Light-curve analysis is presented in Figures 1 and 2. Figure 1 shows that the total flux could be well fit with a sine function with $5700 \pm 300$ day periodicity. Figure 2 shows Lomb-Scargle analysis fluxes for each $1000 \mathrm{~km} \mathrm{~s}^{-1}$ radial velocity bin of $\mathrm{H} \alpha$ with significance level above $90 \%$. The resultant values ranged from $P \approx 5000$ to 6000 days (see Figure 2).

\subsection{Periodical Variations in Light-curves and Possible Mechanisms}

The detection of significant periodicity is a major result of this study. The analysis of the light curves showed a periodicity of nearly 16 years, which is in agreement with previous studies (of 16 year periodicity) obtained (using different methods) for a much earlier monitoring period, covering few earlier cycles (see Oknyanskij et al. 1978).

There are several possible sources of periodicity. Apart from the orbital period ( $T_{\mathrm{P}} \approx 5.5 r_{16}^{-3 / 2} M_{7}^{-1 / 2} \mathrm{yr}$ ), relevant timescales include the timescale associated with geodetic precession $T_{P} \approx$ $2 \times 10^{4} r_{16}^{-5 / 2}(M / m) M_{7}^{-3 / 2} \mathrm{yr}$ (i.e., precession of the $\mathrm{BH}$ spin around the total angular momentum of the system; Begelman et al. 1980) and accretion disk precession due to a second massive $\mathrm{BH} T_{P, \mathrm{AD}} \approx 23 \times r_{16}^{3}(M / m)^{1 / 2} m_{6}^{-1 / 2} R_{d, 16}^{-3 / 2} \cos ^{-1} \theta_{0} \mathrm{yr}$,

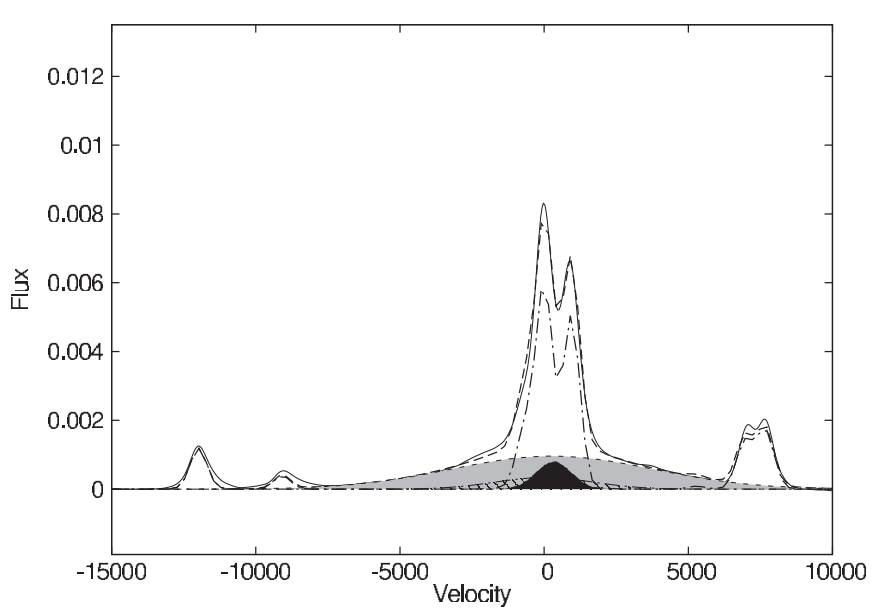

Figure 9. Same as for Figure 8, but for the epoch MJD 52016.36, with the bump component positioned on the central part of the spectrum.

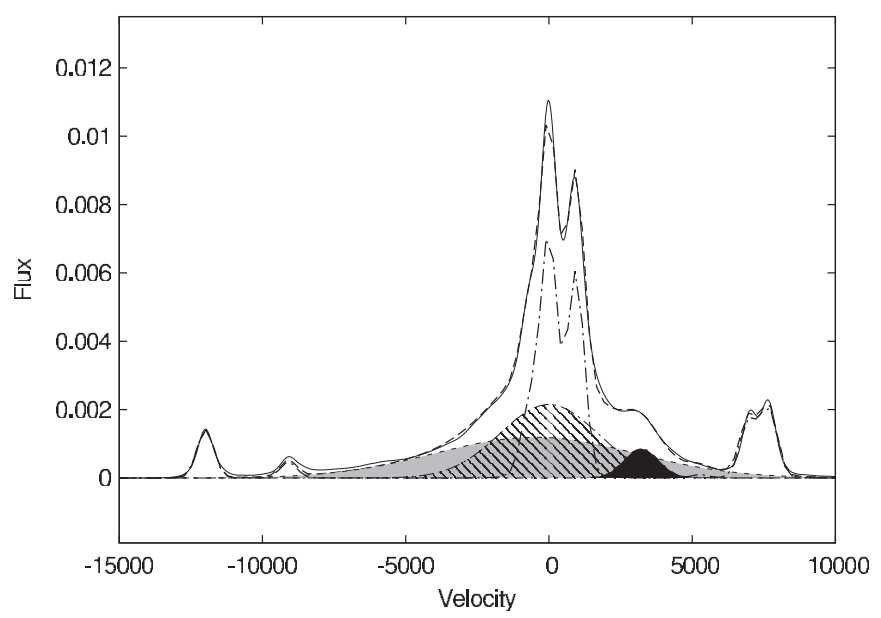

Figure 10. Same as for Figure 8, but for the epoch MJD 52621, with the bump component positioned on the red part of the spectrum.

where $R_{d}$ is the accretion disk radius (Katz 1997). Measured parameters for NGC 4151 imply that the geodetic precession is $T_{P} \sim 10^{5} \mathrm{yr}$. Accretion disk precession is expected in any AGN hosting a binary $\mathrm{BH}$, and $T_{P, \mathrm{AD}}$ is much lower than the value estimated for geodetic precession. However, for NGC 4151, $T_{P, \mathrm{AD}} \approx 250 R_{d, 16}^{-3 / 2} \cos ^{-1} \theta_{0}$ is still significantly longer than the derived period. A third possibility is that radiation force induces a "self-warping" of the accretion disk (obviating the necessity for a second $\mathrm{BH}$ ). In this case, the period would be (Pringle 1996) $T_{\mathrm{P} \text {,warp }} \approx 13.6 \times\left(M_{\text {ion.gas, }-2}\right) M_{7}^{1 / 2} R_{\mathrm{d}, 10^{3} \mathrm{R}_{\mathrm{g}}}^{1 / 2} L_{44}^{-1} \mathrm{yr}$. The self-warping timescale is consistent with the observed $P$ and could, in principle, reinforce periodic behavior also involving continuum fluxes.

\subsection{A Sub-parsec Supermassive Black Hole Binary}

We analyzed radial velocity curves for the VBC and bump components because we noticed large variations of their velocity shifts. Figure 4 indicates that the radial velocity curve can be well described with Keplerian orbits in a binary system.

The derived orbit is eccentric $(e=0.42)$ with a period of $P=$ 5776 days and longitude of pericenter $\omega \approx 95^{\circ}$. We note that orbital precession would be expected but we did not take it into account, since the predicted $\delta \Phi<1^{\circ}$ per period in the Schwarzschild metric. Using Kepler's laws, we estimated the 
semimajor axes: $a_{1} \sin i=0.002 \mathrm{pc}$ and $a_{2} \sin i=0.008 \mathrm{pc}$, and masses: $m_{1} \sin ^{3} i=3 \times 10^{7} M_{\odot}$ and $m_{2} \sin ^{3} i=8.5 \times 10^{6} M_{\odot}$ (where $i$ is the inclination of the orbital plane). These results are in good agreement with the corresponding theoretical results obtained using numerical simulations (Bogdanović et al. 2008) that showed simulations (including the emission line profiles) of an eccentric Keplerian system with a 15.7 year period, at $0.01 \mathrm{pc}$ distance, with similar total mass as found here. We also estimated semimajor axes and masses for different inclination angles and found that an inclination of about $65^{\circ}$ yields semimajor axes of $0.0024 \mathrm{pc}$ and $0.0094 \mathrm{pc}$, while the masses of each component are $4.4 \times 10^{7} M_{\odot}$ and $1.2 \times 10^{7} M_{\odot}$. Our mass estimate is in good agreement with the values obtained from other methods, e.g., reverberation mapping (Onken et al. 2007). The derived separation of two supermassive black holes (SMBHs) of about $0.01 \mathrm{pc}$ is expected from theoretical models for the evolution of binary SMBHs (Milosavljević \& Merritt 2001). The derived period is in agreement with previous studies of optical variability in NGC 4151 (Oknyanskij et al. 1978; Oknyanskij \& Lyuty 2007; Guo et al. 2006), which indicate the existence of previous cycles (see Oknyanskij et al. 1978).

If this model is correct, then gravitational radiation should lead to coalescence (Misner et al. 1973) in $\sim 10^{8}$ yr. The evolution of a BBH system through merging is a topic of much recent research (see Merritt \& Milosavljević 2005 for an exhaustive review). Regardless of the evolutionary stages governed by stellar dynamics, there will be a final stage when dissipation of orbital energy is due to emission of gravitational radiation. When the binary separation is on subparsec scales, coalescence timescales $\propto M^{-3}$ imply that such binary configurations could be relatively long lived in low-mass AGNs, if other mechanisms do not intervene (e.g., Hayasaki et al. 2008). Systems like NGC 4151, with a central SMBH and a (or perhaps, even more than one) much less massive satellite, could be common even if they are hard to find.

Considering the relatively large uncertainty in radial velocity for the $\mathrm{VBC}$, the mass ratio could be different from what we have derived. In the most extreme case, we could be observing some cloud of gas (Gillessen 2012) spiraling down toward the center of attraction, but then we should not expect a very long lifetime for such a cloud due to dissipation, making this scenario less probable, since several 16 year cycles are already reported (Oknyanskij et al. 1978; Oknyanskij \& Lyuty 2007; Guo et al. 2006 , including the result of this paper). If the secondary object is compact but with a mass much smaller than the primary component, then its orbit would be defined with the bump component only, implying a distance of about $0.01 \mathrm{pc}$ and the same period (about 15.9 years and eccentricity of about 0.4 ).

\section{DISCUSSION}

If we assume that a second massive $\mathrm{BH}$ is indeed present, then the pretty large value of eccentricity suggests an analogy with OJ 287 (Valtonen \& Ciprini 2012), where its orbital motion is assumed to be an explanation of its flux variation. Orbital motion of SMBHs has been recently reported in 3C 66B (Sudou et al. 2003) and earlier in 3C390.3 (Gaskell 1996). We are however far from the extreme conditions proposed for OJ 287, a source with a $\mathrm{BH}$ mass estimated to be $\sim 500$ times larger than the one of NGC 4151. As a consequence, we expect a small pericenter precession. Energy loss due to gravitational radiation is very small if compared to OJ 287 . Nevertheless, this system is among those where further evolution is governed by gravitational losses. Since these systems are expected to occur when the binary separation roughly corresponds to the size of the BLR, extensive monitoring may reveal other cases (we remark that the observational coverage of NGC 4151 is almost unique).

If the component separation in a binary $\mathrm{SMBH}$ system is sub-parsec, i.e., on the same order or smaller than the expected radius of the BLR, we have justification for a CBC fixed to the rest frame as derived from the redshift of the narrow lines. This implies that the orbit of the binary SMBH system is most probably located within the BLR region. The estimated orbital velocities $\left(v=v_{r} \cdot \sin ^{-1} i \cdot \sin ^{-1} \phi\right.$, where $i$ represents inclination and $\phi$ the orbital phase) are above $4000 \mathrm{~km} \mathrm{~s}^{-1}$ for the bump and around $1000 \mathrm{~km} \mathrm{~s}^{-1}$ for the VBC for the quadrature phase (where $\sin \phi=1$ and the radial component is highest toward the observer). These are much larger than the typical speed of sound (typically of the order of $10-100 \mathrm{~km} \mathrm{~s}^{-1}$ ) expected for such conditions (Bogdanović et al. 2008; Dopita 1995; Mayer et al. 2007). Therefore, one would expect them to produce shock waves in the BLR (Shi et al. 2012; Artymowicz \& Lubow 1996; Mayer et al. 2007). The epoch with maximum total flux (see Figure 1, bottom), in the MJD interval between 50000 and 51000, corresponds to the epoch when the bump (secondary) component is approaching the observer with supersonic velocity, producing shock waves in the direction of the observer (see Figure 4), while a smaller flux maximum that follows afterward (MJD between 52000 and 53000) corresponds to the epoch when the approaching shock wave is produced by the VBC. The epochs where the fluxes are at minimum (Figure 4) correspond to the phase when the shock waves are propagating perpendicular to the line-of-sight direction (conjunction phase). This is consistent with previous results (Shapovalova et al. 2008): non-radiatively heated emission is needed to explain an excess of line emission with respect to the case of pure photoionization during the epoch of the flux maximum (MJD between 50000 and 51000). Shocks can provide a suitable heating mechanism.

In other scenarios, involving self-warping and flares, one would expect circular concentric orbits with sinusoidally shaped radial velocities. This differs from the radial velocity curves in Figure 4 that correspond to the more eccentric Keplerian orbits. Also, the simulated light and radial velocity curves of an elliptical disk, single spiral arm, or fragmented spiral arm presented in Lewis et al. (2010) could not describe well the shape of the light and radial velocity curves observed in NGC 4151.

One of the alternative explanations for peaks or shoulders seen in the broad $\mathrm{H} \alpha$ profiles could be the emission of an accretion disk. To test such a possibility, we tried to fit the Chen \& Halpern (1989) model of accretion disk into broad line profiles from different epochs, in such a way that the peak of the bump would be fitted with one of two peaks from the disk profile. We found that it could be possible to fit an accretion disk model in most of the profiles, but always needing the additional components. For example, we could fit the disk profile into the core of the line in such a way that the bump component would be covered with one of the peaks from the disk. In that case, the result for the inclination parameter is very small (around $15^{\circ}$ ), which is not that likely of an explanation due to the much higher inclination of jets, around $60^{\circ}-70^{\circ}$ (see Das et al. 2005; Kraemer et al. 2001). The variations in the observed profiles could be explained by assuming that the emission lines originate in the precessing accretion ring, or precessing elliptical accretion ring (Storchi-Bergmann et al. 1997), with the variations of the width of the inner radius of the emitting ring, or with the shift of the disk profile (so the moving bump would be fitted with 
one of the peaks from the disk profile). In all of these cases, it was necessary to involve additional components (e.g., VBC and $\mathrm{CBC}$ ). If the wider disk profile were used to fit the far wings, then the corresponding inclination would be around $45^{\circ}$, covering the far wings of the profiles, as in the $\mathrm{VBC}$ in our model, resulting in a similar behavior to the VBC. This will include the need for the CBC and a bump component and with radial velocity curves similar to those in our simplified model with three Gaussians. We found cases with a higher red peak than blue that could not be explained with the circular disk model, but could be explained with the elliptical disk model (Eracleous et al. 1995). For this alternative model, its precession could explain its rising and moving peaks. All alternative explanations must be consistent with the obtained periodicity (see Section 3.1). Some other alternative explanations could be the orbital motion of a flare or a bright spot (see, for e.g., Newman et al. 1997), selfwarping of the accretion disk (Pringle 1996), precession of an eccentric circular and elliptical accretion disk, circular disk with spiral arm (see, for e.g., Lewis et al. 2010), the precession of a bipolar outflow (see, for e.g., Crenshaw \& Kraemer 2007), and an inhomogeneous disk (see, for e.g., Eracleous et al. 1995). For most of these scenarios, we should expect a more sinusoidal-like behavior of radial velocity curves (see, for e.g., Newman et al. 1997; Storchi-Bergmann et al. 1997; Lewis et al. 2010), which differs from Figure 4. For these alternative models, more tests should be made to prove or disprove any of them.

The radial velocity curve is immediately consistent with orbital motion as we are able to fit it with an eccentric orbit. The idea of orbital motion seems the most plausible. It is a consequence of the favorable value of the argument of the periastron (i.e., a fortunate coincidence) that we are able to see a sudden change in the radial velocity. It is however what is expected and observed in many binaries with highly eccentric orbits.

\section{CONCLUSION}

We have analyzed the shape and flux variations in the $\mathrm{H} \alpha$ light curve for the Seyfert galaxy NGC 4151 using observations spanning 20 years. We also studied variations in different parts of the line profile. Lomb-Scargle spectral analysis of these light curves suggest variations that can be satisfactorily fit with sine functions and indicating a periodicity of about 5700 days for the total flux variation and around 5800 days for most of the $1000 \mathrm{~km} \mathrm{~s}^{-1} \mathrm{H} \alpha$ intervals. Analysis of radial velocity curves for $\mathrm{H} \alpha$ emission line components (VBC and bump) reveal evidence for orbital motion consistent with the signature of a sub-parsec scale SMBH system with an orbital period of about 5780 days.

In this scenario, periodic variations in the optical spectra of NGC 4151 could be generated by supersonic motions of the components through an extended BLR. Maxima in the flux variations correspond to phases when a shock wave is generated toward the observer. This mechanism is also able to explain the detected outbursts in the optical fluxes of emission lines and the continuum and is in agreement with reported shock heating during the period around the light curve maximum (see Shapovalova et al. 2008), as well as the similarity of its X-ray spectra to BH binary spectra in the hard state (Lubinski et al. 2010).

This interpretation is consistent with some previous theoretical results (Bogdanović et al. 2008; Mayer et al. 2007, 2010; Milosavljević \& Merritt 2001) and would support the idea of $\mathrm{BH}$ mass growth by major merger rather than by slower accretion processes. If the phenomenology outlined by our analysis is indeed general for all similar AGNs, then an important question arises: Is binarity a necessary condition for AGN ignition? Support for such an assumption could be related to changes in spectral type (Sy1-Sy1.5-Sy2) manifested by NGC 4151. The activity type of the galaxy changes from Sy1 to Sy1.5 when it is most active (Shapovalova et al. 2008) and to Sy2 at its deep minimum phase, as it was in 1984 (Penston \& Perez 1984; Lyutyj et al. 1984). Different spectral types may be possible only at a certain phase of orbital motion in the binary SMBH. This opens the question of whether the different activity types of active galaxies correspond to different orbital phases of such systems, and whether the binarity is a necessary condition for the activity switch-on. Further monitoring is needed to assess the persistence (or disappearance) of the orbiting components. If our results would be confirmed by future monitoring campaigns or analysis of different sets of data, then NGC 4151 might become an important candidate for gravitational wave detection in the future.

This research is part of projects 176003 "Gravitation and the large scale structure of the Universe" and 176001 "Astrophysical spectroscopy of extragalactic objects" supported by the Ministry of Education and Science of the Republic of Serbia, contract P08-FQM-4205 from La Junta de Andalucia and also grants N09-02-01136a and 12-02-01237a supported by RFBR. We also thank Samir Salim and Giovanni La Mura for constructive discussions.

\section{REFERENCES}

Artymowicz, P., \& Lubow, S. H. 1996, ApJ, 467, L77

Begelman, M. C., Blandford, R. D., \& Rees, M. J. 1980, Nature, 287, 307

Bogdanović, T., Smith, B. D., Sigurdsson, S., \& Eracleous, M. 2008, ApJS, 174,455

Bon, E., Popović, L. Č., Gavrilović, N., La Mura, G., \& Mediavilla, E. 2009, MNRAS, 400, 924

Bon, E., Popović, L. Č., Ilić, D., \& Mediavilla, E. 2006, New Astron. Rev., 50,716

Chen, K., \& Halpern, J. P. 1989, ApJ, 344, 115

Chuvaev, K. K., Oknyanskij, V. L., \& Lyuty, V. M. 2008, Izv. Kr. Astrof. Obs., 104, 109

Crenshaw, D. M., \& Kraemer, S. B. 2007, ApJ, 659, 250

Das, V., Crenshaw, D. M., Hutchings, J. B., et al. 2005, AJ, 130, 945

Dopita, M. A. 1995, Ap\&SS, 233, 215

Eracleous, M., Boroson, T. A., Halpern, J. P., \& Liu, J. 2012, ApJS, 201, 23

Eracleous, M., Livio, M., Halpern, J. P., \& Storchi-Bergmann, T. 1995, ApJ, 438,610

Gaskell, C. M. 1996, ApJ, 464, 107

Gaskell, C. M. 2009, New Astron. Rev., 55, 140

Gaskell, C. M., \& Klimek, E. S. 2003, Astron. Astrophys. Trans., 22, 661

Gillessen, S., Genzel, R., Fritz, T. K., et al. 2012, Nature, 481, 51

Guo, D., Tao, J., \& Qian, B. 2006, PASJ, 58, 503

Hayasaki, K., Mineshige, S., \& Ho, L. C. 2008, ApJ, 682, 1134

Ho, L. C., Filippenko, A. V., \& Sargent, W. L. 1995, ApJS, 98, 477

Kaspi, S., Maoz, D., Netzer, H., et al. 1996, ApJ, 470, 336

Katz, J. I. 1997, ApJ, 478, 527

Komossa, S. 2006, Mem. Soc. Astron. Ital., 77, 733

Kraemer, S. B., Crenshaw, D. M., Hutchings, J. B., et al. 2001, ApJ, 551,671

Kraemer, S. B., Schmitt, H. R., \& Crenshaw, D. M. 2008, ApJ, 679, 1128

Lewis, K. T., Eracleous, M., \& Storchi-Bergmann, T. 2010, ApJS, 187, 416

Lomb, N. R. 1976, Ap\&SS, 39, 447

Longo, G., Vio, R., Paura, P., Provenzale, A., \& Rifatto, A. 1996, A\&A, 312,424

Lubinski, P., Zdziarski, A. A., Walter, R., et al. 2010, MNRAS, 408, 1851

Lyutyj, V. M., Oknyanskij, V. L., \& Chuvaev, K. K. 1984, Sov. Astron. Lett., 10,335

Malkov, Yu. F., Pronik, V. I., \& Sergeev, S. G. 1997, A\&A, 324, 904

Marziani, P., Sulentic, J. W., Negrete, C. A., et al. 2010, MNRAS, 409, 1033

Mayer, L., Kazantzidis, S., Escala, A., \& Callegari, S. 2010, Nature, 466, 26

Mayer, L., Kazantzidis, S., Madau, P., et al. 2007, Science, 316, 1874 
Merritt, D., \& Milosavljević, M. 2005, Living Rev. Relativ., 8, 8

Milosavljević, M., \& Merritt, D. 2001, ApJ, 563, 34

Misner, C. W., Thorne, K. S., \& Wheeler, J. A. 1973, in Gravitation (San Francisco, CA: Freeman), 988

Mundell, C. G., Pedlar, A., Shone, D. L., \& Robinson, A. G. 1999, MNRAS, 304, 481

Mundell, C. G., Wrobel, J. M., Pedlar, A., \& Gallimore, J. F. 2003, ApJ, 583,192

Newman, J. A., Eracleous, M., Filippenko, A. V., \& Halpern, J. P. 1997, ApJ, 485,570

Onken, C. A., Valluri, M., Peterson, B., et al. 2007, ApJ, 670, 105

Oknyanskij, V., \& Lyuty, V. 2007, Perem. Zvezdy Prilozhenie, 7, 28

Oknyanskij, V. L. 1978, Perem. Zvezdy, 21, 71

Pacholczyk, A. G., Penning, W. R., Ferguson, D. H., Lubart, N. D., \& Turnshek, D. 1983, Astrophys. Lett., 23, 225

Penston, M. V., \& Perez, E. 1984, MNRAS, 211, 33

Popović, L. Č. 2012, New Astron. Rev., 56, 74

Press, W. H., Teukolsky, S. A., Vetterling, W. T., \& Flannery, B. P. 1996, Numerical Recipes in Fortran 77: The Art of Scientific Computing (2nd ed.; Cambridge: Cambridge Univ. Press)

Pringle, J. E. 1996, MNRAS, 281, 357
Rainer, W. 1988, Velocity 1.3, A Program to Compute Radial Velocity Curves for Spectroscopic Binary Stars, Determine Best-fit Solutions for Measured Radial Velocity Data, and Plot the Results, http://www.ibiblio.org/ pub/Linux/science/astronomy

Scargle, J. D. 1982, ApJ, 263, 835

Sergeev, S. G. 1994, Astron. Zh., 71, 189

Shapovalova, A. I., Popović, L. Č., Burenkov, A. N., et al. 2010, A\&A, 106, 509

Shapovalova, A. I., Popović, L.C., Collin, S., et al. 2008, A\&A, 486, 99

Shen, Y., \& Loeb, A. 2010, ApJ, 725, 249S

Shi, J. M., Krolik, J. H., Lubow, S. H., \& Hawley, J. F. 2012, ApJ, 749, 118

Storchi-Bergmann, T., Eracleous, M., Ruiz, M. T., et al. 1997, ApJ, 489, 87

Sudou, H., Satoru, I., Yasuhiro, M., \& Yoshiaki, T. 2003, Science, 23, 1263

Sulentic, J. W., Zwitter, T., Marziani, P., \& Dultzin-Hacyan, D. 2000, ApJ, 536,5

Tsalmantza, P., Decarli, R., Dotti, M., \& Hogg, D. W. 2011, ApJ, 738, 20

Ulrich, M. H., Altamore, A., Boksenberg, A., et al. 1985, Nature, 313, 747

Ulvestad, J. S., Roy, A. L., Colbert, E. J. M., \& Wilson, A. S. 1998, ApJ, 496, 196

Valtonen, M., \& Ciprini, S. 2012, Mem. Soc. Astron. Ital., 83, 219

Zamfir, S., Sulentic, J. W., Marziani, P., \& Dultzin, D. 2010, MNRAS, 403, 1759 